\title{
PERTURBAÇÕES DO SONO EM ADULTOS/ IDOSOS HOSPITALIZADOS
}

\author{
Rosa Martins ${ }^{1}$ \\ Ana Marisa Correia ${ }^{2}$ \\ Ana Andrade \\ Sofia Campos ${ }^{3}$
}

\begin{abstract}
Resumo: Contexto: O sono é um bem precioso e indispensável a um bom equilíbrio. $O$ processo de envelhecimento ocasiona perturbações que se refletem tanto na quantidade como na qualidade do sono, sendo estas alterações agravadas em situações de hospitalização.

Objetivos: Avaliar perturbações do sono dos utentes adultos/idosos internados e analisar relação com variáveis sociodemográficas, clínicas e fadiga crónica.

Metodologia: Trata-se de um estudo transversal, descritivo e correlacional, do tipo quantitativo. Para o efeito foi aplicado um formulário a 60 utentes, internados, que incluía variáveis sociodemográficas, uma Escala de Fadiga Crónica e o Índice de Qualidade do Sono de Pittsburg (IQSP).

Resultados: A amostra é maioritariamente constituída por utentes do sexo masculino, casados, com 75 anos e analfabetos. Realizam programa de reabilitação diariamente entre 16 a 30 minutos e apenas algumas vezes se sentem aptos para o concretizar. A maioria $(55,00 \%)$ sente-se mais ativo no período da tarde, dorme em media 6,8 horas, acorda muitas vezes e mais cedo do que o pretendido. A fadiga crónica está presente na grande maioria dos inquiridos, sentindo estes que precisam de dormir mais do que normalmente dormem.

Conclusões: A maioria dos participantes (96,70\%) apresenta perturbações de sono no internamento, sendo estas mais graves nos utentes com índices mais elevados de fadiga crónica, com mais idade, nas mulheres e nos viúvos.
\end{abstract}

\footnotetext{
${ }^{1}$ Professora Coordenadora da Escola Superior de Saúde do Instituto Politécnico de Viseu. Email: rmmartins.viseu@gmail.com

${ }^{2}$ Hospital da Nossa Senhora da Assunção de Seia

3 Escola Superior de Saúde do Instituto Politécnico de Viseu. Email: sofiamargaridacampos@gmail.com
} 
Palavras-chave: Perturbações do sono, utentes, idosos, hospitalização.

Title: SLEEP QUALITY IN HOSPITALIZED ADULTS / ELDERLY

Abstract: Background: Sleep is precious and indispensable to a good balance. The aging process causes disturbances which reflect both the quantity and quality of sleep, being that these changes are aggravated in hospital situations.

Objectives: To evaluate sleep disorders of adults / hospitalized elderly patients and analyze the relationship with sociodemographic variables, clinical and chronic fatigue.

Methodology: This is a cross-sectional, descriptive and correlational study, of the quantitative type. For this purpose, a questionnaire was applied to 60 users, hospitalized, that included sociodemographic variables, a Chronic Fatigue Scale and the Sleep Quality Index of Pittsburgh (PSQI).

Results: The sample is mostly made up of male users, married, 75 years old and illiterate. They have performed their rehabilitation program daily between 16-30 minutes and only a few times do they feel ready to finish. The majority (55.00\%) feel more active at afternoon, they sleep on average 6.8 hours, wake up often and sooner than intended. Chronic fatigue is present in the vast majority of respondents, with these feeling that they need to sleep more than what they normally sleep.

Conclusions: The majority of participants (96.70\%) present sleep disorders during hospitalization, with this being associated to users with higher rates of chronic fatigue, of an older age, women and widows.

Keywords: Sleep Quality, users, elderly, hospitalization.

\section{INTRODUÇÃO}

O sono é uma necessidade fisiológica, que tem como funções biológicas a restauração do organismo e a conservação da energia, permitindo um equilíbrio físico e emocional, que é fundamental à vida (Martins, 2004).

Os estudos desenvolvidos na área do sono têm mostrado que a duração e os padrões do sono sofrem alterações significativas no decurso da ontogenia humana, havendo evidências de que a estrutura cíclica e a distribuição das diferentes fases do sono variam com a idade. Os valores máximos normalmente, estão ligados à primeira infância, contudo a partir da puberdade inicia-se um ligeiro declínio que vai progredindo na vida adulta e se agrava na velhice. Na opinião de Manable et al. (2000), com o 
passar dos anos, a pessoa idosa sofre alterações no seu padrão de sono, tanto em latência e eficiência como na duração, constituindo este facto um dos maiores e insolúveis problemas desta faixa etária.

Os mecanismos do ritmo vigília/sono na opinião de Martins (data, cit. por Mugeiro, 2011) são extremamente complexos e ainda não foram totalmente esclarecidos, contudo, trata-se de um ritmo fundamental e estreitamente relacionado com os outros ritmos quotidianos que compreende vários aspetos: tem caráter reversível, podendo do sono passar-se à vigília e vice-versa; fenómeno cíclico, pois sono e vigília alternam-se ciclicamente de modo periódico e regular em ciclos de 24 horas; durante o sono há ausência quase total de movimento; o sono implica um aumento ao limiar para responder a estímulos ambientais/externos; produz uma atividade psicológica denominada por "sonho" e finalmente durante o sono, ocorrem várias modificações tanto na atividade cerebral como noutras atividades psicofisiológicas.

A descoberta do electroencefalograma (EEG) possibilitou a demonstração das alterações da atividade elétrica cerebral, que ocorrem durante o sono, mostrando que não se trata de um estado homogéneo, mas sim de um fenómeno em que existem dois estados distintos: o sono REM e o sono não REM. A denominação de sono lento (NREM) provém do facto de o traçado electroencefalográfico mostrar uma lentificação progressiva, que aumenta à medida que o sono se torna mais profundo, ocorrendo uma atividade parassimpática dominante, com miose, diminuição da sudação, da frequência cardíaca, da tensão arterial, da temperatura, do consumo de $\mathrm{O} 2$ e do tónus muscular (Rente \& Pimentel, 2004).

O sono paradoxal (sono REM) é assim denominado devido ao fenómeno que contrasta movimentos rápidos oculares e atonia muscular generalizada, predominando a atividade simpática, com grande variabilidade autonómica, nomeadamente da frequência cardíaca e respiratória. Nesta fase existe ainda um aumento da temperatura, da sudação e do consumo de $\mathrm{O}^{2}$ (Nércio, 2010).

Em termos funcionais, o sono REM tem sido relacionado com a restauração das funções cognitivas (ideia que é reforçada pelo aumento do sono REM intra-uterino e durante a infância, em que há um grande desenvolvimento cerebral), enquanto que o sono NREM é considerado um restaurador das funções orgânicas (durante o sono NREM ocorre um aumento de anabolismo proteico de algumas hormonas como a do crescimento e da testosterona). Esta teoria - da Restauração do Organismo - explica por que motivo os sintomas da privação de sono 
parcial, contínua e total, são ao mesmo tempo de caráter físico e intelectual. As evidências científicas têm demonstrado que o sono desenvolve funções primordiais na preservação de energia e restauração de competências físicas e mentais do organismo, capacitando-o de forma saudável e competente para as tarefas que decorrem durante a vigília. Mas para que isto ocorra, é necessário dormir um número de horas considerado suficiente (Seixas, 2009).

A questão da durabilidade do sono não tem reunido consensos quanto à média diária necessária, contudo esta variabilidade existe, quer quanto ao número médio de horas necessárias de sono, quer quanto à estrutura do próprio sono ao longo da vida, facto que tem sido atribuído à diversidade das diferenças individuais e das características ontogénicas. Para o autor supracitado, o número total de horas de sono necessárias, a partir dos sessenta e cinco anos, situa-se entre seis e oito horas, contudo há que considerar que a passagem dos anos não afeta somente a quantidade, mas também a própria qualidade do sono.

$\mathrm{Na}$ verdade, os estudos levados a cabo por Pinto (2007), Oliveira (2008), e Mugeiro (2011), têm posto a descoberto a elevada prevalência dos transtornos do sono em diferentes culturas e grupos de adultos/idosos. Estima-se que cerca de um terço da população apresentará algum tipo de disfunção do sono durante a sua vida, e em Portugal, os distúrbios de sono afetam cerca de $30 \%$ da população, sendo os indivíduos mais afetados sobretudo idosos e mulheres (Paiva \& Penzel, 2011). Diz-nos Pinto (2007) que os idosos têm menos capacidade de dormir de forma contínua e, como tal, o seu sono é mais frequentemente interrompido por despertares noturnos ou seja mais fragmentado e menos profundo. Em consequência aceita-se que os efeitos da idade sobre a capacidade de dormir tenha profundas implicações na manutenção do humor, da atenção e da eficiência cognitiva do idoso, bem como o aparecimento ou o agravar de outras ocorrências como sejam o aumento de utilização de hipnóticos e o cansaço fácil. Estas alterações objetivas no padrão do sono acompanham-se de uma sensação subjetiva de dormir menos profundamente, acontecendo variações em pessoas sem transtornos psíquicos ou físicos, o que nos indica que o sono tem naturalmente uma evolução temporal (Duarte, 2008). Um sono adequado é definido pela combinação de três factores: Latência; Eficiência e Duração. De acordo com a Academia Americana da Medicina do Sono, para um sono adequado, a pessoa deve levar quinze minutos para adormecer, estar adormecido pelo menos $85 \%$ do tempo total que passa na cama e dormir por um período de tempo não inferior a sete horas. Um indivíduo 
apresenta privação, quando o seu padrão de sono sofre alterações na quantidade e na qualidade, ou seja, o sono é afetado, pelo menos, na sua eficiência e duração. Os sinais e sintomas que daí resultam variam consoante a idade, ambiente, personalidade e motivação. Em consequência, as mudanças comportamentais que poderão ocorrer são a desorientação, desassossego, irritabilidade e uma atitude anti-social, podendo progredir para um comportamento mais agressivo e até psicótico se a privação permanecer (Silva, 2006).

As perturbações do sono são, como já referenciámos, frequentes nos adultos/idosos, contudo em situação de hospitalização estas perturbações agravam, podendo o indivíduo apresentar privação, fragmentação e até dessincronização (Manable et al, 2000). De facto durante a hospitalização, o utente está muitas vezes sujeito à privação do sono, por múltiplas circunstâncias, sendo acordado várias vezes e/ou mantido acordado por diferentes razões. O stress causado pelo processo de hospitalização, pode configurar-se como uma experiência potencialmente traumática que afasta o indivíduo de sua rotina diária e motiva em muitas situações um confronto com a dor e a limitação física, além de aflorar sentimentos indesejáveis. Os estudos realizados com o objetivo de avaliar as perturbações do sono em clientes hospitalizados, concluíram globalmente que o sono em contexto hospitalar é mais pobre comparativamente com o sono que os utentes desenvolviam em casa e que as perturbações que ocorrem estão ligadas essencialmente a fatores ambientais e a situações stressantes.

\section{MATERIAL E MÉTODOS}

A metodologia consiste em descrever de forma sistemática a estrutura e as etapas de uma pesquisa. Trata-se portanto de um estudo quantitativo, do tipo não experimental, transversal e descritivo-correlacional.

As questões de investigação que este estudo pretende explorar são: (1) "será que o sono dos adultos/idosos internados apresentam boa qualidade/perturbações? (2) "Em que medida a qualidade do sono destes utentes se correlaciona com as variáveis sociodemográficas, fadiga e processos de reabilitação?". Os objetivos, decorrentes das questões enunciadas, consistem em identificar a qualidade do sono que é percecionada pelos Participantes e analisar fatores influentes no sono avaliado.

Optámos por uma amostra do tipo não probabilístico por conveniência, constituída por 60 utentes internados nos serviços de 
Medicina e Unidades de Convalescença de um hospital da zona Centro do País. A maioria dos utentes são do sexo masculino (51,7\%), casados (53,3\%), na faixa etária acima dos 75 anos, com idade média de 73,1 anos a variar entre um mínimo de 49 anos e um máximo de 94 anos, a viverem maioritariamente com a família $(60,0 \%)$ em meio rural $(65,0 \%)$.

Os critérios de inclusão estabelecidos para o estudo foram: ser utentes internados em serviços de Medicina e Unidades de Convalescença; possuir capacidade de resposta verbal ou escrita; realizarem diariamente programas de reabilitação.

Todos os procedimentos devem ser efetuados segundo uma rigorosa conduta ética respeitando os princípios da beneficência, da justiça e de um respeito integral pela dignidade humana: assim foi dirigido ofício com pedido de autorização para a aplicação do instrumento de colheita de dados (ICD) ao Presidente do Conselho de Administração da U.L.S da Guarda, E.P.E, onde era referenciado o responsável pela pesquisa, explicitados os objetivos e finalidades do estudo. Para além, do pedido formal à instituição foi apresentado a todos os participantes o documento de consentimento informado, a garantia de anonimato e confidencialidade dos dados recolhidos e ainda a liberdade de em qualquer altura poderem desistir se assim o desejassem. Depois de ser dada a autorização procedeu-se à fase de recolha de dados que foi realizada entre os meses de outubro de 2012, a fevereiro de 2013.

A escolha dos métodos de colheita de dados depende das variáveis estudadas, da sua operacionalização, das questões de investigação/hipóteses e do desenho do estudo delineado. No nosso caso optámos pela utilização de um formulário por nós construído e pela aplicação de escalas elaboradas por outros investigadores e consequentemente, já traduzidas e testadas na população portuguesa. $\mathrm{O}$ instrumento de colheita de dados (ICD) deste estudo, inclui questões de caracterização sociodemográfica, clínica, de perceção dos utentes sobre o seu sono, Escala de Fadiga Crónica (EFC) traduzida e adaptada para a população portuguesa por Silva, Azevedo \& Dias (1995) e o Índice de Qualidade do Sono de Pittsburgh (IQSP) adaptado e validado para português por Alessandra Bertolazi, em 2008, que avalia a qualidade do sono das pessoas no último mês.

O tratamento estatístico da presente investigação foi efectuado através do programa Statiscal Package Social Science versão 19.0 para o Windows e Word Microsoft. 


\section{RESULTADOS}

Verificámos que a idade dos elementos da amostra, oscila entre os 49 e os 94 anos, com uma média que ronda os 73,10 anos. O maior valor percentual dos utentes $(43,30 \%)$ possui idades superiores a 75 anos, seguindo-se os que se situam na faixa etária entre 66 e 75 anos $(35,00 \%)$. Verificámos ainda, que os sujeitos do sexo masculino, eram ligeiramente mais novos que as mulheres (71,03 e 75,31 anos). Estes dados estão em consonância com os publicitados pelo INE dos Censos 2011, uma vez "que a esperança média de vida para a população portuguesa se situa nos 79,20 anos, não se processando a longevidade de forma equitativa, uma vez que no século XXI vai persistir uma diferença de 6 anos, favorável à mulher podendo estas viverem em média 82,05 anos (superior á dos homens que é de 76,14). Verificamos ainda, que a relação entre a idade e a qualidade do sono só se verificou aos níveis da eficiência habitual do sono e das perturbações, uma vez que os participantes mais velhos apresentam padrões de sono com menor eficiência habitual e maiores perturbações.

Existe homogeneidade no nosso estudo quanto ao género, uma vez que dos 60 indivíduos que compõem a amostra, 31 são do sexo masculino e 29 do sexo feminino. Estes últimos (elementos do sexo feminino) foram aqueles que apresentaram maiores alterações do sono sobretudo aos níveis da latência do sono, na eficiência habitual e ainda na qualidade total do sono, à semelhança dos resultados de Mugeiro (2011), em que as mulheres em geral expressam maior prevalência de distúrbios de sono quando comparadas com os homens.

O local de residência para $65 \%$ dos participantes no estudo é o meio rural, sendo estes essencialmente mulheres. Estes resultados não nos surpreenderam dado que a maioria dos utentes da região geográfica onde está inserida a nossa instituição (distrito da Guarda) provêm essencialmente do meio rural. A associação entre local de residência e perturbações do sono mostra que são independentes (sem associação significativa) apesar dos valores das ordenações médias apontarem para perturbações do sono mais graves nos participantes do meio urbano.

O estado civil prevalente é o de "casado" (53,30\%), seguindo-se os viúvos $(41,70 \%)$, os divorciados/separados $(1,70 \%)$ e apenas $3,30 \%$ solteiros. Verificámos ainda que os homens eram essencialmente casados $(74,20 \%)$, enquanto a maioria das mulheres $(62,10 \%)$ era viúva. Estes resultados reforçam as tendências demográficas publicitadas pelo INE (2011), onde a viuvez é essencialmente feminina devido aos efeitos da 
sobremortalidade masculina, aos efeitos dos fluxos migratórios e ainda ao facto dos homens depois de enviuvarem optarem pela reconstituição familiar (Mugeiro, 2011). Constatámos ainda, que os viúvos, solteiros e divorciados apresentavam mais distúrbios do sono do que os casados e/ou em união de facto, localizam-se estes fundamentalmente na eficiência habitual do sono. O estudo de Trindade-Filho, Carvalho, \& Gomes (2010) revelou dados similares uma vez que existia " uma maior prevalência de queixas do sono, como é o exemplo da insónia, nos indivíduos separados e viúvos. De facto há autores que demonstram existir uma grande associação entre solidão e insónia, que segundo eles é um aspecto comum em indivíduos separados.

A composição do agregado familiar é uma variável importante na medida em que é na família que se definem os padrões de atendimento e comportamento dos seus membros. O afeto entre os familiares, a constância dos mesmos e o senso de obrigação que permeia esses mesmos vínculos garantem ao indivíduo trocas constantes de suporte instrumental, psicológico e emocional. Assim, 60,0\% dos participantes vive com a família, $20 \%$ vive sozinho e os restantes $20 \%$ encontram-se institucionalizados. A análise por género mostra que embora os utentes de ambos os sexos coabitem maioritariamente com a família, existe uma percentagem mais elevada de mulheres $(27 \%)$ a morar sozinhas. Não encontrámos relações estatísticas significativas entre o agregado familiar e a qualidade do sono; contudo Gaillard (1996) aponta as diferenças de personalidade e as formas de convivência, como fatores interferentes na duração do sono. Por exemplo, pessoas que habitualmente dormem pouco (menos de seis horas), em sua opinião, são mais ativas, trabalhadoras, mais extrovertidas, mas com tendências hipomaníacas. Já as pessoas que dormem muito (dez horas ou mais) são frequentemente mais ansiosas, introvertidas, e depressivas.

Os baixos níveis de escolaridade observados na nossa amostra corroboram os estudos realizados por Martins (2004), Branco, Nogueira e Dias (2011), ao referirem que a maioria da população idosa portuguesa possui baixos níveis de instrução. Na verdade, $43,30 \%$ dos nossos utentes não sabe ler nem escrever, $40 \%$ possuem apenas o $1^{\circ}$ ciclo de estudos e $16,70 \%$ possuía o $2^{\circ}$ Ciclo. Constatamos ainda que as mulheres possuem predominantemente menores habilitações literárias do que os homens, o que é igualmente compreensível se tivermos em conta a moldura sociocultural que por largos anos prevaleceu na sociedade portuguesa. A única dimensão onde encontramos significância estatística $(\mathrm{p}=0,011)$ foi nas perturbações do sono, onde os participantes com o $1^{\circ}$ ciclo de estudos 
apresentam maiores dificuldades. Constatamos ainda que os sujeitos analfabetos têm médias mais altas (ou seja, pior sono) nas dimensões duração do sono; eficiência habitual do sono e disfunção diurna. Sabemos que a formação académica propícia aos indivíduos a aquisição de conhecimentos e consequentes comportamentos que podem favorecer estilos de vida saudáveis. A boa organização da higiene do sono, é um dos exemplos que poderia trazer, não só melhoria na qualidade de sono como na qualidade de vida e promoção da saúde desta faixa etária.

Os programas de reabilitação regulares têm-se revelado fundamentais na tipologia de doentes destas unidades e por isso os participantes do nosso estudo realizam na totalidade programas de reabilitação. O tempo gasto em cada sessão pode ser variável, sendo variável também os seus efeitos. No nosso estudo, constatamos que o tempo despendido no programa de reabilitação não interfere na qualidade do sono (sem significância estatística). Não obstante os sujeitos que apenas gastam entre 0 e 15mn no programa de reabilitação, são também aqueles que genericamente apresentam mais perturbações nas diferentes dimensões e qualidade total do sono. Já os participantes que gastam entre 30 a 60 minutos no programa possuem pior qualidade do sono apenas na dimensão duração. Relacionando estes dois constructos Oliveira, Rossini, \& Reimão, (2008) realçam a pertinência de diferentes intervenções junto dos utentes/idosos, entre as quais a dos enfermeiros de reabilitação, no sentido de prevenir o envelhecimento patológico e promover condições pessoais e mudanças sócio ambientais que permitam reequilibrar a harmonia entre o individuo as suas funções biológicas e o ambiente que o rodeia.

Poder-se-ia questionar se uma pior aptidão para realizar o programa de reabilitação corresponderia a uma pior qualidade de sono ou viceversa, mas a falta de estudos que suportem este pressuposto não nos permitem formular teorias válidas sob o ponto de vista científico. O nosso estudo aponta para inexistência de relação entre a qualidade de sono e o programa de reabilitação, apesar dos valores das ordenações médias revelarem que os sujeitos que raramente se sentem aptos para realizar o programa de reabilitação, apresentam pior qualidade subjetiva do sono, pior latência do sono, mais perturbações, usam mais medicação, apresentam mais disfunção diurna e apresentam globalmente pior qualidade total do sono. Contrariamente, os que referiram sentir-se aptos frequentemente apresentam mais distúrbios que os outros apenas nas dimensões duração do sono e eficiência habitual do sono. A explicação para estes factos, em nosso entender, poderão estar relacionados com o 
avanço da idade, onde ocorrem perdas na duração, manutenção e qualidade do sono e ainda com a hospitalização, onde tudo isto se agrava. As perturbações do sono afetam os indivíduos de modo significativo tanto a nível físico, psicológico, intelectual (no aproveitamento das capacidades cognitivas), nas manifestações comportamentais e na estabilidade emocional e mesmo nas perturbações de desenvolvimento como hiperatividade, défice de atenção, défice cognitivo e depressão). Assim, os utentes que não obtêm o descanso e o sono necessários (e nós constámos que $36,70 \%$ dos inquiridos referem precisar de dormir muito mais tempo do que aquele que normalmente dormem) terão repercussões negativas no seu processo terapêutico.

Segundo o levantamento norte americano efetuado por Zimmer e Lima (2004, cit. por Júnior, 2007), a fadiga constitui o nono sintoma mais comum em Atenção Primária à Saúde (APS): o presente estudo robustece estes achados, uma vez que constatamos que $65,00 \%$ revela fadiga ligeira, 23,30\% manifesta fadiga moderada e 3,30\% apresenta fadiga acentuada. A análise por género mostra que a maioria $(62,10 \%)$ das mulheres apresenta fadiga ligeira, paralelamente aos sujeitos do sexo masculino $(67,70 \%)$, contudo a fadiga está mais presente nos elementos do sexo feminino. Verificámos ainda, que existe uma forte associação entre a fadiga e a qualidade do sono dos participantes, sendo esta altamente significativa na maioria das dimensões e qualidade total do sono. Os participantes com fadiga moderada são os que apresentam mais perturbações aos níveis das dimensões qualidade subjetiva do sono e uso de medicação hipnótica. Na verdade, a privação do sono parcial tem como efeitos a diminuição do desempenho psicomotor, lapsos de atenção e dificuldades de concentração, redução da memória para acontecimentos recentes, tempos de reação prolongados, mau humor, sensação de fadiga, irritabilidade e até estados confusionais (Paiva, \& Penzel, 2008; Seixas,2009).

Por fim, e centrando a nossa atenção no objectivo fundamental deste estudo, verificámos que apenas 3,30\% dos participantes percepciona ter sono com boa qualidade. Contrariamente, a grande maioria $(96,70 \%)$ considera ter alguma perturbação de sono classificando-o como sendo de má qualidade, com maior relevância no caso das mulheres. São dados que reforçam o paradigma nacional, visto que em Portugal, os distúrbios de sono afetam $30 \%$ da população, sendo os idosos e as mulheres os mais afetados. Estudos conduzidos por Veras (2009) demonstram que o aumento nos despertares precoces e a dificuldade em manter o sono relacionados com o envelhecimento podem derivar de uma incapacidade 
de manter o sono numa fase específica do ritmo circadiano. Como tal, a privação dessa estabilidade dá origem à dessincronização interna e tem como consequência danos para a saúde do adulto/idoso. A dessincronização externa (como sejam os fatores ambientais ligados ao internamento), por sua vez, associa-se às mudanças no padrão de sono, o que se deve à diminuição progressiva dos estímulos sociais, que atuam como sincronizadores. Os fatores que interferem nessa dessincronização são: a insuficiência qualitativa e quantitativa desses estímulos; a redução da acuidade dos órgãos dos sentidos, ou seja, dos recetores dos estímulos ambientais e a dificuldade do sistema de temporização circadiana em adequar os ritmos de acordo com os estímulos sociais. A par do que fica dito, as perturbações do sono são ainda mais frequentes aquando da hospitalização (devido a fatores intrínsecos como a doença, dor e alterações de humor, e a fatores extrínsecos, como a luminosidade, ruído, medicação e as rotinas e intervenções de enfermagem) que impedem um sono adequado, levando o indivíduo a apresentar privação, fragmentação e dessincronização (Silva, 2006).

\section{CONCLUSÕES/ IMPLICAÇÕES PARA A PRÁTICA}

A pertinência do tema do nosso estudo perece-nos inegável, pelas razões amplamente apontadas nos pontos anteriores, contudo este pressuposto sai reforçado se validarmos o paradigma atual de que a idade é um fator determinante na fisiologia do sono. $\mathrm{Na}$ verdade e independentemente das mudanças próprias do envelhecimento, são múltiplos os fatores que contribuem para estas alterações do sono dos utentes o que explica a variedade de respostas as questões formuladas. Existem variadíssimos problemas, que embora não sejam específicos do adulto/idoso, têm especial relevância em pessoas com maior idade em situação de internamento, pelas suas repercussões no sono: inadaptação perante situações emocionais perturbadoras, maus hábitos de sono, perturbações psiquiátricas afetivas, doenças orgânicas, consumo de medicamentos (psicotrópicos ou não), agitação noturna ou quedas. Os problemas descritos são cruciais nos resultados da reabilitação destes indivíduos, uma vez que concorrem para resultados menos eficientes do processo, prolongando-os no tempo e levando muitos deles a exaustão.

As questões referidas sobre as perturbações do sono nos Idosos reforçam a necessidade em investigar esta temática nos diferentes contextos, uma vez que os resultados dos estudos vão divergindo de acordo com as condições sociais económicas e culturais dos grupos. A 
reflexão sobre os resultados deste estudo em confronto com os conhecimentos anteriores, mostram que continuam em aberto muitas questões que suscitam novos avanços e novas investigações. Assim da análise dos resultados obtidos e tendo em atenção os objetivos propostos inicialmente, concluímos que:

$\checkmark$ A amostra estudada é maioritariamente masculina (51,6\%), situando-se a média de idades nos 73 anos; São predominantemente casados $(51,30 \%)$, sem habilitações académicas $(43,30 \%)$, a residir em meio rural $(65,00 \%)$ e a viver com a família $(60,00 \%)$. Os que vivem sozinhos e/ou estão institucionalizados são essencialmente mulheres.

$\checkmark$ Todos os inquiridos realizam programa de reabilitação no período da manhã, com um tempo médio de 16 a 30 minutos, sentem-se mais ativos no período da tarde e talvez por isso, somente algumas vezes se sentem aptos para realizar as sessões.

$\checkmark$ Constámos que $36,70 \%$ dos inquiridos precisa de dormir muito mais do que normalmente dorme e acorda muitas vezes mais cedo do que o pretendido. Em termos médios, dormem 6,8 horas por noite, referem ter sempre dificuldades em adormecer e o tempo que precisam para começar a funcionar bem depois de uma noite de sono, oscila entre 11 a 20 minutos $(46,70 \%)$;

$\checkmark$ A fadiga crónica está muito presente neste grupo (apenas 8,3\% não a refere), uma vez $92,7 \%$ dos utentes apresenta fadiga que varia entre ligeira a acentuada.

$\checkmark$ A percepção de $96,70 \%$ dos participantes é que tem uma má qualidade de sono, sendo esta mais referida pelos elementos do sexo feminino.

$\checkmark$ As correlações encontradas mostram diferenças estatísticas significativas entre a idade e a qualidade do sono sobretudo nas dimensões eficiência habitual do sono e as perturbações do sono em que os mais velhos são os mais afetados; entre o sexo e a qualidade do sono nas dimensões latência do sono; eficiência habitual do sono e qualidade total, sendo neste caso mais afectadas as mulheres; os viúvos, solteiros e divorciados apresentam mais perturbações do sono do que os casados e/ou em união de facto, essencialmente na dimensão eficiência habitual do sono; as habilitações literárias interferem na qualidade do sono (perturbações do sono), sendo os participantes com o $1^{\circ}$ ciclo os que apresentam pior qualidade do sono; por fim verificamos que existem diferenças estatísticas altamente significativas entre todas as dimensões e qualidade do sono total e a fadiga, inferindo-se que quanto maior for a fadiga pior é a qualidade do sono dos participantes. 
$\checkmark$ Contrariamente, o local de residência, a coabitação, a aptidão e o tempo para os programas de reabilitação revelaram-se independentes da qualidade do sono nestes participantes, dado não existirem diferenças estatísticas significativas.

Temos consciência que este estudo constitui uma jornada de esforço, determinação mas também uma fonte de aprendizagem. Os desafios, as dificuldades e o poder aliciante e fascinante da investigação sobre o sono dos adultos/idosos internados falaram mais alto dando motivação para apontar novos caminhos a desenvolver em futuras investigação.

\section{BIBLIOGRAFIA}

Branco, M. J., Nogueira, A. P. J., \& Dias, C. M. (2011). Mocecos: Uma observação dos cidadãos idosos no princípio do século XXI. Lisboa: ONSA.

Bertolazi, A. N. (2008). Tradução, adaptação cultural e validação de dois instrumentos de avaliação do sono: escala de sonolência de Epworth e índice de qualidade do sono de Pittsburgh. [Em linha]. Porto Alegre: Rio Grande do Sul: Universidade Federal do Rio Grande do Sul., 2008. 93 p. Dissertação de Mestrado. [Consult.25 Set. 2011]. Disponível em WWW:

<URL:http://www.lume.ufrgs.br/bitstream/handle/10183/14041/00065 3543.pdf? sequence $=1>$.

Duarte, J. C. (2008). Privação do sono, rendimento escolar e equilibrio psico-afectivo na adolescência (Dissertação de doutoramento, Universidade do Porto). Acedido em data 3 de Agosto de 2011 http://hdl.handle.net/10216/19371.

Gaillard, J. M. (1996). Perturbações do sono: Clínica e terapêutica. Lisboa: Roche Farmacêutica Química. Lda, s.d.

Junior, P. P. P. (2007). O sono normal. In P. L. M. Albernaz (Org.), Durma bem, viva melhor (pp. 23-26). São Paulo: MG Editores.

Manable, K.,

Matsui, T., Yamaya, M., SatoNakagawa, T., Okamura, N., Arai, H., \& Sasaki H. (2000). Sleep patterns and mortality among elderly patients in a geriatric hospital. Gerontology, 46(6), 318-322.

Martins, R. (2004). Qualidade de vida dos idosos da Região de Viseu. Badajoz: [s.d.], (Dissertação de doutoramento não publicada). Universidade da Extremadura, Badajoz, Espanha. 
Mugeiro, M. J. C. (2011). Qualidade do sono nos Idosos. Viseu (Dissertação de mestrado, Instituto Politécnico de Viseu). Acedido em data 5 de junho de 2012 http://repositorio.ipv.pt/handle/10400.19/1658.

Nércio, T. R. (2010). Qualidade do sono e hábitos de estudo em jovens institucionalizados. (Dissertação de mestrado, Universidade Fernando Pessoa, Porto). Acedido em 27 de Junho de 2011 http://hdl.handle.net/10284/1462.

Oliveira, J. C, Rossini, S., \& Reimão, R. (2008). O sono no idoso: Mudanças na arquitectura do sono e insónia. In R. Reimão, L. E. L. Ribeiro do Valle, \& S. Rossini, Segredos do sono (pp.49-57). São Paulo: Tecmedd.

Paiva, T., \& Penzel, T. (2011). Centro de medicina do sono: Manual prático. Lisboa: Lidel.

Portugal, Instituto Nacional de Estatística (2011). Censos: Resultados definitivos. Acedido em 9 de julho de 2012 $\mathrm{http}: / /$ censos.ine.pt/xportal/xmain?xpid=CENSOS\&xpgid=censos 201 1_apresentacao

Pinto, C. (2007). O Sono no idoso, quando dormir é um problema. Arquivo: Mais Saúde. Acedido em 8 de Setembro de 2011 de http://www.medicosdeportugal.saude.sapo.pt/utentes/senior/o_sono_n o_idoso_quando_dormir_e_um_problema/3.

Rente, P., \& Pimentel, T. (2004). A patologia do sono. Lisboa: LIDEL.

Seixas, M. P. (2009). Avaliação da qualidade do sono na adolescência: Implicações para a saúde física e mental. (Dissertação de mestrado, Universidade Fernando Pessoa, Porto). Acedido em 23 de agosto de 2012 http://hdl.handle.net/10284/1256.

Silva, C. F. (2006). Fundamentos teóricos e aplicações da cronobiologia. Psicologia, Teoria Investigação e Prática, (5) 2, 253-265.

Silva, Carlos, F.; Azevedo, M. H.; Dias, M. R. (1995) - Estudo padronizado do trabalho por turnos. Versão Portuguesa do SSI. Psychologica ${ }^{\circ}$ 13, 1995, p. 27-36.

Trindade-Filho, E. M., Carvalho, L. N. A., \& Gomes, E. O. (2010). Estudo da qualidade do sono na população adulta de Maceió. Neurobiologia, 73 (1), 93-97. Acedido em 21 de Setembro de 2011 http://www.neurobiologia.org/ex_2010/10_EUCLIDESqualidade_de_ sono_pronto(OK).pdf.

Veras, R. (2009). Population aging today: Demands, challenges and innovations. Revista de Saúde Pública, 43(3), 548-554. Acedido em 20 de Julho de 2011 http://www.scielo.br/pdf/rsp/v43n3/en_224.pdf 